Check for updates

Cite this: Nanoscale Adv., 2020, 2 , 2693

Received 28th February 2020 Accepted 14th March 2020

DOI: $10.1039 / d 0 n a 00164 c$

rsc.li/nanoscale-advances

\section{Au nano-urchins enabled localized surface plasmon resonance sensing of beta amyloid fibrillation $\dagger$}

\author{
Radhika V. Nair, (D) ${ }^{a}$ Pae Jian Yi, (D) ${ }^{a}$ Parasuraman Padmanabhan, (DD ${ }^{b}$ \\ Balázs Gulyás (iD b and V. M. Murukeshan (iD *a
}

\begin{abstract}
Early stage detection of neurodegenerative diseases such as Alzheimer's disease (AD) is of utmost importance, as it has become one of the leading causes of death of millions of people. The gradual intellectual decline in $A D$ patients is an outcome of fibrillation of amyloid beta 1-42 $\left(A \beta_{1-42}\right)$ peptides in the brain. In this paper, we present localized surface plasmon resonance (LSPR) based sensing of $A \beta_{1-42}$ fibrillation using Au nano-urchins. Strongly localized field confinement at the spiky nanostructures of nano-urchin surfaces enables them to detect very low concentrations of $A \beta_{1-42}$. In addition, the LSPR peak of Au nano-urchins, which is very sensitive to ambient conditions, shows significant responses at different fibrillation stages of $A \beta_{1-42}$. Reduction in LSPR peak intensity with an increase in the fibrillation is chosen as the sensing parameter here. This paper in this context provides LSPR based highly sensitive, label-free and real-time sensing of $A \beta_{1-42}$ fibrillation that is highly advantageous compared to the existing techniques which require binding additives or fluorescent biomarkers.
\end{abstract}

Alzheimer's disease (AD) is a neurodegenerative disorder caused due to the cerebral accumulation of neurotoxic amyloid beta $(A \beta)$ oligomers., ${ }^{1,2}$ Rapid aggregation of $A \beta_{1-42}$, which is a protein abundant in the central nervous system of humans, results in the formation of soluble oligomers known as amyloid $\beta$-derived diffusible ligands (ADDLs). ${ }^{3}$ Proteolytic activities of $\beta$ and $\gamma$ secretase on amyloid proteins lead to the formation of $\mathrm{A} \beta_{1-42}$ fibrils which trigger the onset of $\mathrm{AD} .{ }^{4}$ Clinical symptoms of $\mathrm{AD}$ such as memory loss and personality changes appear only in the progressed stage of $A \beta_{1-42}$ fibrillation. ${ }^{5}$ At this stage the disease becomes acute and medication may not have a positive impact on the patient. Hence it is crucial to detect $A \beta_{1-42}$

${ }^{a}$ Center for Optical and Laser Engineering (COLE), School of Mechanical and Aerospace Engineering, Nanyang Technological University (NTU), 639798, Singapore. E-mail: mmurukeshan@ntu.edu.sg

${ }^{b}$ Lee Kong Chian School of Medicine, Nanyang Technological University, 639798, Singapore

$\dagger$ Electronic supplementary information (ESI) available. See DOI: 10.1039/dona00164c fibrillation at a very early stage. Recently various biosensing and bioimaging techniques have been developed for the detection of such chronic diseases. ${ }^{6,7}$ Computerized tomography (CT) scan, positron emission tomography (PET) and magnetic resonance imaging (MRI) are the brain imaging techniques currently used to detect various biochemical changes in the brain. ${ }^{8-10}$ These approaches suffer from the disadvantage that they work only after the progression of the disease. Enzyme-linked immunosorbent assay and optical dye (Thioflavin $\mathrm{T}$ and Congo Red) binding are the other techniques used for the study of kinetics of $\mathrm{A} \beta_{1-42}$ fibrillation. ${ }^{11,12}$ These techniques also have disadvantages such as non-specific binding, low stability and crossreactivity. ${ }^{13}$ As a result, these techniques are susceptible to falsepositive results. Fluorescence resonance energy transfer (FRET) is also frequently used for the study of amyloid beta kinetics. ${ }^{\mathbf{1 4 , 1 5}}$ The efficiency of FRET depends hugely on the distance between the binding sites and on the covalent or mechanical linking of proteins with the donor and acceptor molecules. ${ }^{16}$ Hence FRET requires proper understanding of the covalent modification of proteins and careful examination which makes the technique time consuming and complex.

Various optical techniques using photonic crystals, quantum dots, fluorescence correlation spectroscopy etc. have been reported for the real time monitoring and imaging of beta amyloid fibrillation. ${ }^{\mathbf{1 7 - 1 9}}$ Nanoscale structures are reported to be highly significant in biosensing applications. ${ }^{\mathbf{2 0 , 2 1}}$ Recently, metal nanoparticles have become extremely significant in biosensing research owing to the generation of surface plasmon resonances (SPR). ${ }^{22-26}$ Due to the high sensitivity of SPR towards local environmental changes, it serves as a feasible candidate for biosensing and imaging applications. ${ }^{27,28}$ Apart from high sensitivity, SPR based sensing provides real-time and label-free sensing of biomolecules. ${ }^{29,30}$ Metal nanoparticles can be used directly in the assay without any binding additive or fluorescent biomarkers. Metal nanoparticles have been used by various groups for sensing different fibrillation stages of $\mathrm{A} \beta_{1-42} \cdot{ }^{31-33}$ Metal nanostructures with patterned nano features are also used for various bio applications. ${ }^{34}$ In this paper we present the 
study of fibrillation kinetics of $\mathrm{A} \beta_{1-42}$ using $\mathrm{Au}$ nano-urchins. $\mathrm{Au}$ nano-urchins have various advantages over spherical metal nanoparticles. The sharp spiky morphology of urchins provides larger surface area for the binding of $\mathrm{A} \beta_{1-42}$ monomers than spherical particles. ${ }^{35}$ In addition, the sharp tips of the spiky structures over the surface provide stronger localized field enhancement giving rise to better sensitivity towards trace level sensing. Various applications of Au nano-urchins such as in electrochemical biosensors for the detection of genetically modified DNA, melamine sensors, gas sensors and SERS sensors have been reported so far. ${ }^{36-41}$ Here, we introduce a novel application of Au nano-urchins where each stage of $\mathrm{A} \beta_{1-}$ ${ }_{42}$ fibrillation can be sensed based on the intensity of localized surface plasmon resonance (LSPR). Au urchins are observed to be sensitive to nano-molar concentration of $A \beta_{1-42}$ while metal nanospheres are sensitive to millimolar or micromolar concentrations of $A \beta_{1-42}{ }^{42}$

We performed finite difference time domain (FDTD) simulations to prove the localized electric field enhancement in $\mathrm{Au}$ nano-urchins compared to Au nano-spheres. A mesh size of $0.2 \mathrm{~nm}$ was used in simulations. Au nano-spheres and nanourchins of diameter $100 \mathrm{~nm}$ were modelled using Lumerical software. Separation between the particles is $5 \mathrm{~nm}$ in all the simulations. These nanostructures were then excited with a Gaussian source of $633 \mathrm{~nm}$ wavelength. Frequency domain field monitors were used for the study of electric field distribution around the structures. Fig. 1(a) and (b) show the electric field distribution around $\mathrm{Au}$ nano-spheres and nano-urchins, respectively. Electric field enhancement $(|E|)$ of around 9 times is observed for $\mathrm{Au}$ nano-urchins (22.8) compared to $\mathrm{Au}$ nano-spheres (2.63). Hotspots with enhanced electric field intensity are observed to be generated at the vicinity of spiky nanostructures which are highly useful in LSPR based sensing applications.

Based on the results obtained from FDTD simulations, we experimentally examined the potential of $\mathrm{Au}$ nano-urchins in amyloid beta $\left(A \beta_{1-42}\right)$ peptide fibrillation sensing which is the biomarker for Alzheimer's disease. Au nano-urchins of diameter $100 \mathrm{~nm}\left(3.84 \times 10^{9}\right.$ particles per $\mathrm{ml}$, in $0.1 \mathrm{mM}$ PBS $)$ were purchased from Sigma Aldrich. The optical absorption spectrum of Au nano-urchins is shown in Fig. 2(a). The LSPR peak of $\mathrm{Au}$ nano-urchins is observed at $650 \mathrm{~nm}$. The transmission electron microscopy (TEM) image of Au nano-urchins of (a)

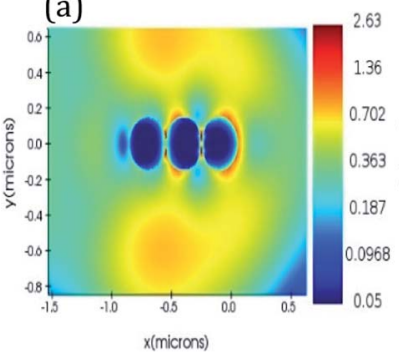

(b)

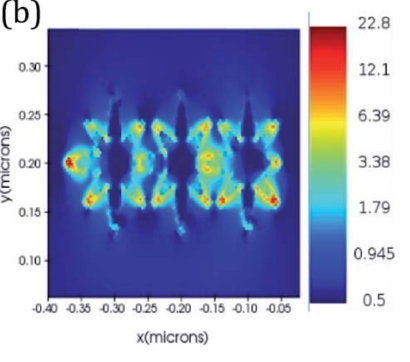

Fig. 1 FDTD simulations of electric field enhancement in (a) Au nanospheres (diameter $=100 \mathrm{~nm}$ ) and (b) Au nano-urchins (diameter $=100 \mathrm{~nm}$ ) at an excitation wavelength of $633 \mathrm{~nm}$.
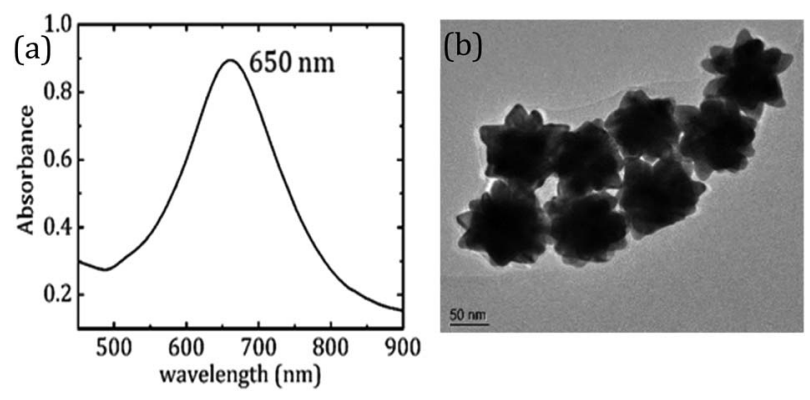

Fig. 2 (a) Optical absorption spectrum of Au nano-urchins and (b) TEM image of Au nano-urchins.

diameter $100 \mathrm{~nm}$ is shown in Fig. 2(b). Spiky nano protrusions over the surface of Au urchins are clearly evident from TEM images.

$\mathrm{A} \beta_{1-42}$ peptide was purchased from Abcam (purity $>95 \%$ ). $\mathrm{A} \beta_{1-42}$ solution was prepared by mixing $1 \mathrm{mg}$ of $\mathrm{A} \beta_{1-42}$ in $100 \mu \mathrm{l}$ of $\mathrm{NaOH}$. Further, the solution is diluted to a concentration of $1 \mathrm{mg} \mathrm{ml}^{-1}$ in phosphate buffered saline (PBS). Samples were then aliquoted to $100 \mu \mathrm{l}$ parts and were stored at $-20{ }^{\circ} \mathrm{C}$. Fibrillation kinetics of $\mathrm{A} \beta_{1-42}$ was first tested using the Thioflavin $\mathrm{T}$ (ThT) assay which is the standard method used to confirm the activity of $\mathrm{A} \beta_{1-42}$ peptide. ${ }^{31}$ ThT emission gets enhanced when it is bound to $A \beta_{1-42}$ fibrils. This emission feature of ThT is used for the detection of various fibrillation stages of $\mathrm{A} \beta_{1-42}$. To study the kinetics of $\mathrm{A} \beta_{1-42}, 100 \mu \mathrm{M}$ ThT stock solution is diluted to $1 \mu \mathrm{M}$ with de-ionised water. $100 \mu \mathrm{l}$ of $\mathrm{A} \beta_{1-42}$ solution is then mixed with $1 \mu \mathrm{M}$ ThT solution. Emission studies were performed at an excitation wavelength of $410 \mathrm{~nm}$ which corresponds to the absorption peak of ThT. Fluorescence intensity was measured every 10 minutes. Fig. 3 shows the emission plot of ThT at various incubation times of $A \beta_{1-42}$. The growth process of $A \beta_{1-42}$ was observed to show three phases lag phase, growth phase and steady phase. A slight increase in emission intensity until 40 minutes indicates the self-assembly of $\mathrm{A} \beta_{1-42}$ monomers during the lag phase. After 40 minutes a sharp increase in emission intensity is observed which is due to the binding of a large number of $\mathrm{A} \beta_{1-42}$ fibrils to ThT during the growth phase. After 60 minutes, fibrillation reaches a steady phase beyond which there is no notable increase in ThT

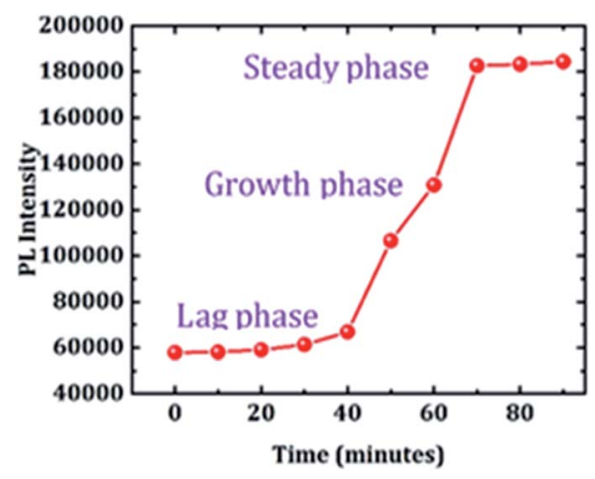

Fig. 3 Kinetics study of $A \beta_{1-42}$ fibrillation using the ThT assay. 
emission. Though ThT can sense $A \beta_{1-42}$ fibrillation, due to the possible disadvantage of cross-reactivity and non-specific binding, label free LSPR based sensing can be a better option for bio sensing.

Samples for biosensing study were prepared by mixing $50 \mu \mathrm{l}$ of $A \beta_{1-42}$ solution with $500 \mu \mathrm{l}$ of $\mathrm{Au}$ nano-urchins. The solution was further diluted to $1 \mathrm{ml}$ using PBS. The experimental set-up for the study of LSPR is shown in Fig. 4. A stabilized broadband light source ( $360 \mathrm{~nm}$ to $2600 \mathrm{~nm}$ ) is used as the excitation source. An optical fiber is used to couple the source to the focusing lens assembly. Focused light falls on the sample in a cuvette. Transmitted light through the sample is collected using the lens assembly which is coupled to a spectrometer using an optical fiber. Absorbance (LSPR) values are then deducted from the transmission data. It is easy to reconfigure the system according to the requirement. Flexibility of using spectrometers in the required range, and the possibility of changing the focal spot on the sample are the advantages of this method over commercial spectroscopy instruments.

In order to sense the fibrillation stages of $A \beta_{1-42}$, an absorbance study of the sample is performed at various time intervals. Excitation over the range of $400 \mathrm{~nm}$ to $800 \mathrm{~nm}$ is used to monitor the change in absorption of Au nano-urchins $(650 \mathrm{~nm}$ peak) in the presence of $A \beta_{1-42}$. Data are recorded at every 5 minute interval for 90 minutes. Fig. 5(a) shows a change in absorption of $A \beta_{1-42}$ with time. A gradual reduction in Au nanourchin absorbance is observed with the increase of time.

In order to clearly identify the absorption peak of Au nanourchins in PBS, baseline-corrected data are given in Fig. 5(b). Baseline correction is done based on the absorption of PBS and the same buffer solution is used for suspending Au nanourchins and amyloid beta peptides and is used for Fig. 2 as well. Absorbance data of samples at incubation times of $10 \mathrm{~min}$, $60 \mathrm{~min}$ and $90 \mathrm{~min}$ are plotted in Fig. 5(b). The absorbance peak at $650 \mathrm{~nm}$ corresponds to Au nano-urchins and that at $735 \mathrm{~nm}$ corresponds to the absorption of beta amyloid. A decrease in LSPR intensity with time is clearly visible from Fig. 5(b). Absorption enhancement observed in the wavelength range $700 \mathrm{~nm}$ to $800 \mathrm{~nm}$ is attributed to mutual absorption contribution from PBS and $A \beta_{1-42}$ (ESI $\dagger$ ). The absorbance peak intensity at $650 \mathrm{~nm}$ is used as the sensing parameter for the sensing of $A \beta_{1-42}$ fibrillation.

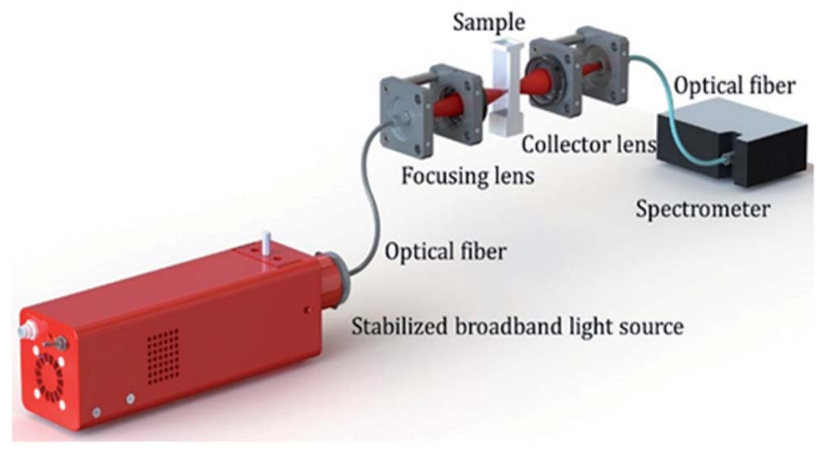

Fig. 4 Experimental set-up for LSPR study.
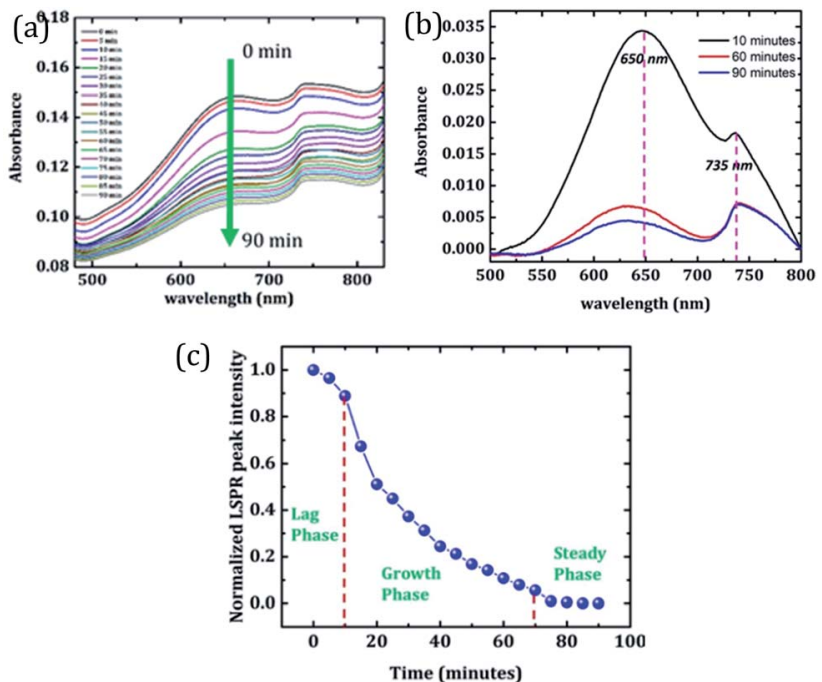

Fig. 5 (a) Absorbance vs. wavelength plots for the Au nano-urchin$A \beta_{1-42}$ sample at various incubation times $(0 \mathrm{~min}$ to $90 \mathrm{~min}$ ), (b) baseline corrected absorbance plot of sample Au nano-urchins-PBS$A \beta_{1-42}$, and (c) LSPR sensing of various fibrillation stages of $A \beta_{1-42}$.

Fig. 5(c) shows a change in $650 \mathrm{~nm}$ peak (LSPR peak) intensity with time. It is observed from the graph that the peak intensity remains almost constant until 10 minutes which indicates the lag phase of $A \beta_{1-42}$. At this stage $A \beta_{1-42}$ monomers slowly start getting aggregated. Since the solution lacks agglomerates, the LSPR intensity of Au urchins is not affected at this stage. A rapid reduction in peak intensity is observed in the time interval from 10 minutes to 70 minutes suggesting the growth phase of $A \beta_{1-42}$. Many $A \beta_{1-42}$ fibrils are formed at this stage and these fibrils tend to bind around Au nano-urchins hindering their plasmonic response. This resulted in the reduction of LSPR peak intensity. As more and more fibrils surround $\mathrm{Au}$ nano-urchins, the LSPR intensity gets rapidly reduced. After 70 minutes, the absorbance values remain constant indicating the steady phase where $A \beta_{1-42}$ fibrillation has almost completed. It is inferred from the results that the LSPR peak of $\mathrm{Au}$ nano-urchins can be used as an efficient sensing tool for the sensing of $A \beta_{1-42}$ fibrillation. LSPR peak intensity changes even at a short interval of 5 minutes clearly suggest the sensitivity of the LSPR peak of Au nano-urchins towards biosensing applications.

According to the literature, the presence of Au nanoparticles may not always cause inhibition or acceleration of beta amyloid peptide aggregation. ${ }^{1,43}$ The interaction between Au nanoparticles and beta amyloid peptides are dependent on the surface charge properties of bare Au nanoparticles which are highly dependent on the surface functionalization of Au nanoparticles. Xiong et al. have reported that a negative surface charge on bare Au nanoparticles results in the lack of coupling between beta amyloid peptides and bare $\mathrm{Au}$ nanoparticles resulting in almost null influence on beta amyloid fibrillation. ${ }^{44}$ In the present work we have used bare Au nano-urchins purchased from Sigma Aldrich, stabilized in PBS buffer solution. There may be a change in the refractive index due to fibrillation of beta amyloid. 
In order to confirm the effect of refractive index, FDTD simulations are performed to study LSPR intensity decay during the fibrillation process. An increase in fibrillation is represented as an increase in effective refractive index in FDTD simulations. $\mathrm{Au}$ nano-urchins are distributed on beta amyloid fibrils in simulations as seen in FESEM images. Electric field calculations are performed between Au nano-urchin spikes and the separation between the spikes is considered as $5 \mathrm{~nm}$ in all simulations. Simulations are performed at an excitation wavelength of $633 \mathrm{~nm}$. Fig. 6(a-g) show the electric field calculations between the spikes for various refractive index values of fibrils (refractive indices from 1.6 to 1.9 in steps of 0.5 representing various fibrillation stages of beta amyloid). A reduction in LSPR field distribution around the urchin spikes is observed with an increase in refractive indices of fibrils and the results agree with the experimental observations.

The experiment is repeated using Au nanospheres of diameter $100 \mathrm{~nm}$ to confirm the better sensitivity of Au nano-urchins towards beta amyloid sensing. The experimental parameters are the same as those for $\mathrm{Au}$ nano-urchins. Fig. 7 shows the comparison between LSPR sensing study of beta amyloid fibrillation at the nano-molar concentration level using Au nano-urchins and $\mathrm{Au}$ nanoparticles. It is observed from the results that the Au nanospheres do not show much change in optical absorption compared to $\mathrm{Au}$ nano-urchins at various fibrillation stages of beta amyloid fibrillation at nano-molar concentration, limiting the low concentration beta amyloid fibrillation sensing capability of Au nanospheres.

Further, to double-check the $\mathrm{A} \beta_{1-42}$ fibril formation, Field Emission Scanning Electron Microscopy (FESEM) images of $\mathrm{A} \beta_{1-42}$ were taken at different incubation times. Radial growth of beta amyloid fibrils is observed in Fig. 8(a-c). Pompom like growth of beta amyloid fibrils has been already reported in the literature. ${ }^{45}$ Fig. 8(a-c) show the FESEM images of $\mathrm{A} \beta_{1-42}$

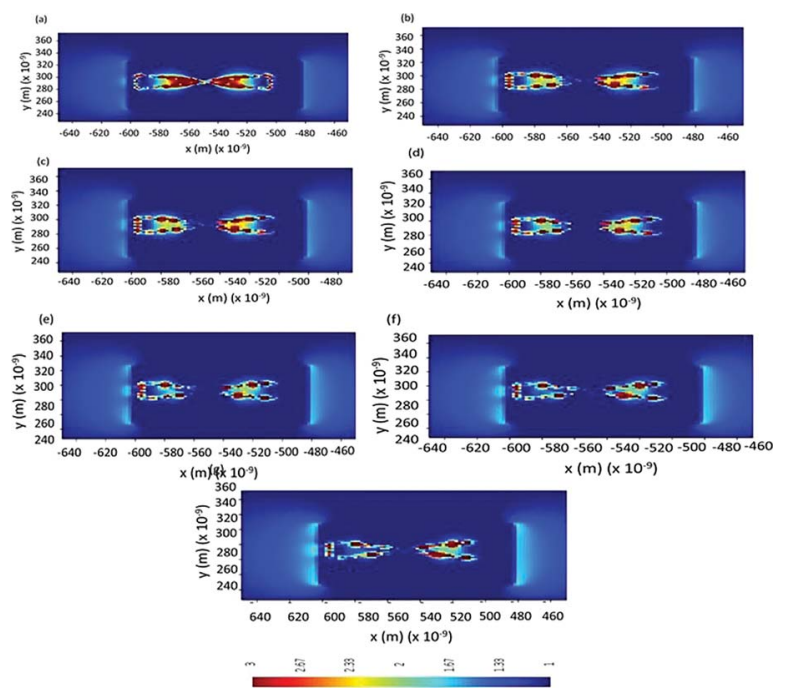

Fig. 6 FDTD simulations of electric field calculations between Au nano-urchin spikes distributed on amyloid beta fibrils with various refractive index values: (a) 1.6, (b) 1.65, (c) 1.7, (d) 1.75, (e) 1.8, (f) 1.85, and (g) 1.9, respectively (approximation for beta amyloid fibrillation).

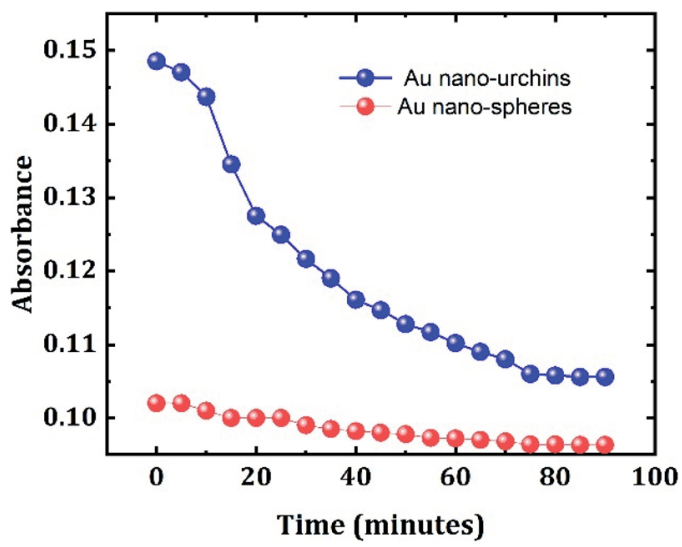

Fig. 7 Comparison between LSPR sensing of beta amyloid fibrillation at the nano-molar concentration level using Au nano-urchins (blue) and Au nano-spheres (red).

mixed with Au nano-urchins for incubation times of 5 minutes (lag phase), 40 minutes (growth phase) and 90 minutes (steady phase), respectively. It is clear from Fig. 8(a) that $A \beta_{1-42}$ fibrils are in the lag phase. A few small fibrils of length around $400 \mathrm{~nm}$ are observed in the lag phase which indicates the beginning of monomer aggregation. Au nano-urchins are observed as a white layer in the background. Nucleation of monomers to fully grown fibrils appears to occur at various regions in Fig. 8(b) indicating the growth phase. From Fig. 8(c) it is inferred that the fibrils are elongated and almost the whole area is covered with fibrils of length $2 \mu \mathrm{m}$ to $3 \mu \mathrm{m}$ indicating the steady phase of $\mathrm{A} \beta_{1-42}$ fibrillation. Au nanourchins are found to be bound at various points on the inner as well as outer surfaces of fibrils which are indicated using red circles in Fig. 8(c). FESEM images confirm the results obtained from $\mathrm{Au}$ urchin based LSPR study.

A major advantage of using Au nano-urchins in beta amyloid fibrillation sensing lies in the peripheral diagnosis of Alzheimer's disease (AD). According to the previous literature, tissue level changes of $\mathrm{AD}$ are detectable years before their clinical expression. ${ }^{\mathbf{4 6}}$ These changes in beta amyloid proteins reflect in the body fluids as these proteins are excreted to different body fluids. There are reports on non-invasive detection of beta amyloid from body fluids such as saliva and blood. ${ }^{47,48}$ Hence peripheral diagnosis of $\mathrm{AD}$ in an non-invasive way is highly significant in early stage detection of beta amyloid fibrillation. In the present work, LSPR sensing with Au nanourchins mainly targets future peripheral diagnostic applications rather than in vivo applications. For the in vivo applications, further optimization of the aspect ratio is required to cross the blood brain barrier. Though the cytotoxicity of $\mathrm{Au}$ nanoparticles is not fully studied, they are observed to be biocompatible in comparison with other metal nanoparticles. $\mathrm{Au}$ nanostructures are also known to induce nanoparticle induced endothelial leakage which will be helpful for the transportation of nanomedicine. ${ }^{49}$ With further optimization of the aspect ratio of Au nano-urchins, these structures may be useful for in vivo applications. 

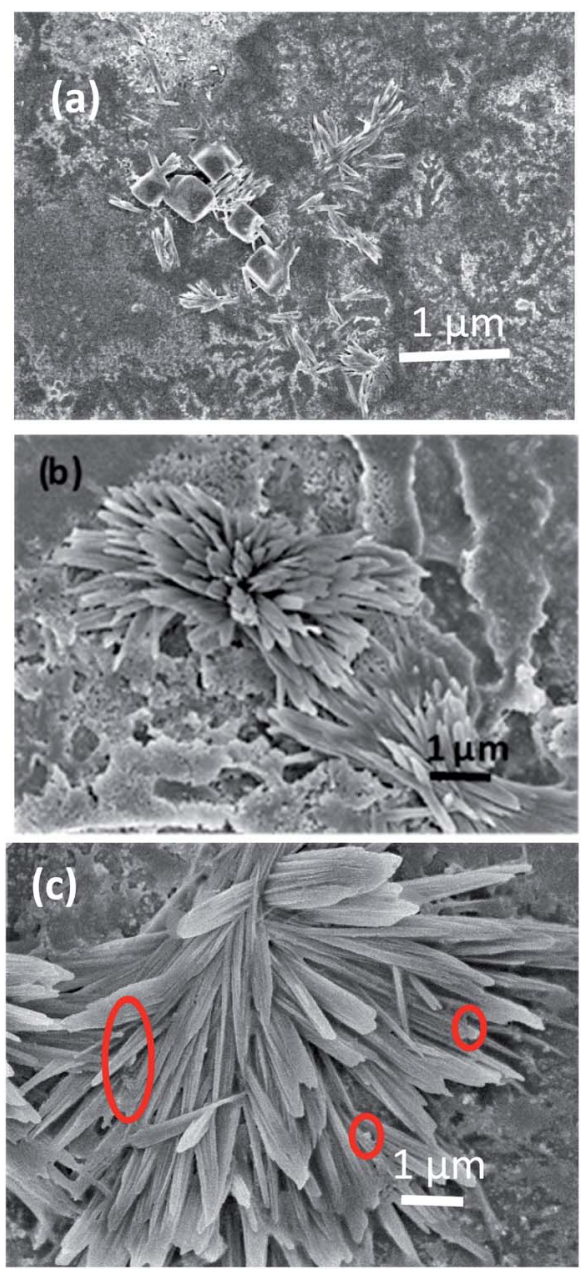

Fig. 8 FESEM images of $A \beta_{1-42}$ mixed with $A u$ nano-urchins for incubation times of 5 minutes (lag phase) (a), 40 minutes (growth phase) (b) and 90 minutes (steady phase) (c); red circles indicate Au nano-urchins attached to $A \beta_{1-42}$ fibrils.

One of the advantages of using Au nano-urchins for beta amyloid fibrillation sensing is the possibility of fibrillation sensing at low concentrations due to the enhanced localized plasmon resonance. Though this paper reports on the nanomolar concentration level sensing as a starting point, there is a scope for further improvement in the sensing concentration by changing the density and thickness of spikes over the surface of $\mathrm{Au}$ nano-urchins which will have a huge influence on the localized SPR field enhancement. Though optical dyes such as ThT and Congo red can give similar information on the fibrillation kinetics of beta amyloid, the concentration for accurate sensing is observed to be higher which can adversely affect the capability of optical dyes in early stage beta amyloid fibrillation sensing. ${ }^{50}$

\section{Conclusions}

In conclusion, we demonstrate LSPR based sensing of $\mathrm{A} \beta_{1-42}$ fibrillation using Au nano-urchins in this paper. The LSPR peak of $\mathrm{Au}$ nano-urchins is observed to be highly sensitive to structural changes of $\mathrm{A} \beta_{1-42}$ during the fibrillation process even at nano-molar concentration. The progress in the fibrillation process is sensed based on the reduction in LSPR peak intensity of $\mathrm{Au}$ nano-urchins. Enhanced localized electric field generation at the spiky structures over Au nano-urchins and high sensitivity of LSPR to ambient conditions enable them to act as an efficient biosensor. We envisage that this work can lead to the realization of highly sensitive, label free and real time sensing of beta amyloid enabling $\mathrm{AD}$ detection.

\section{Conflicts of interest}

There are no conflicts to declare.

\section{Acknowledgements}

The authors acknowledge NTU, COLE-EDB, and Ministry of Education, MoE Singapore (RG 192/17) for financial support.

\section{Notes and references}

1 T. John, A. Gladytz, C. Kubeil, L. L. Martin, H. J. Risselada and B. Abel, Nanoscale, 2018, 10, 20894-20913.

2 X. Yi, C. Feng, S. Hu, H. Li and J. Wang, Analyst, 2016, 141, 331-336.

3 X. Wang, G. Perry, M. A. Smith and X. Zhu, Neurodegener. Dis., 2010, 7, 56-59.

4 J. Nunan and D. H. Small, FEBS Lett., 2000, 483, 6-10.

5 J. Zhao, X. Zhang, C. R. Yonzon, A. J. Hoes and R. P. Van Duyne, Nanomedicine, 2006, 1, 219-228.

6 Y. Xia, M. V. Matham, H. Su, P. Padmanabhan and B. Gulyás, J. Biomed. Nanotechnol., 2016, 12, 1553-1584.

7 S. Padmanabhan, V. K. Shinoj, V. M. Murukeshan and P. Padmanabhan, J. Biomed. Optic., 2010, 15, 017005.

8 P. Scheltens, Dialogues Clin. Neurosci., 2009, 11, 191-199.

9 C. Marcus, E. Mena and R. M. Subramaniam, Clin. Nucl. Med., 2014, 39, 413-426.

10 S. Shokouhi, D. Claassen and W. R. Riddle, J. Alzheimer's Dis. Park., 2014, 4, 2.

11 D. Tes, K. Kratkiewicz, A. Aber, L. Horton, M. Zafar, N. Arafat, A. Fatima and M. R. Avanaki, Biomed. Eng. Comput. Biol., 2018, 9, 117959721878108.

12 L. Song, D. R. Lachno, D. Hanlon, A. Shepro, A. Jeromin, D. Gemani, J. A. Talbot, M. M. Racke, J. L. Dage and R. A. Dean, Alzheimer's Res. Ther., 2016, 8, 1-15.

13 E. I. Yakupova, L. G. Bobyleva, I. M. Vikhlyantsev and A. G. Bobylev, Biosci. Rep., 2019, 39, 1-22.

14 T. Takahashi and H. Mihara, Chem. Commun., 2012, 48, 1568-1570.

15 M. C. Owen, D. Gnutt, M. Gao, S. K. T. S. Wärmländer, J. Jarvet, A. Gräslund, R. Winter, S. Ebbinghaus and B. Strodel, Chem. Soc. Rev., 2019, 48, 3946-3996.

16 B. T. Bajar, E. S. Wang, S. Zhang, M. Z. Lin and J. Chu, Sensors, 2016, 16, 1488.

17 Y. Guan, K. J. Cao, A. Cantlon, K. Elbel, E. A. Theodorakis, D. M. Walsh, J. Yang and J. V. Shah, ACS Chem. Neurosci., 2015, 6, 1503-1508. 
18 M. Yamao, K. Aoki, N. Yukinawa, S. Ishii, M. Matsuda and H. Naoki, PLoS One, 2016, 0164254.

19 S. Santi, V. Musi, E. Descrovi, V. Paeder, J. Di Francesco, L. Hvozdara, P. Van Der Wal, H. A. Lashuel, A. Pastore, R. Neier and H. P. Herzig, ChemPhysChem, 2013, 14, 34763482.

20 V. M. Murukeshan, J. K. Chua, S. K. Tan and Q. Y. Lin, Opt. Express, 2008, 16, 13857.

21 J. K. Chua and V. M. Murukeshan, Phys. Scr., 2009, 80, 1-5.

22 P. Palladino, A. M. Aura and G. Spoto, Anal. Bioanal. Chem., 2016, 408, 849-854.

23 P. P. Vachali, B. Li, A. Bartschi and P. S. Bernstein, Arch. Biochem. Biophys., 2015, 572, 66-72.

24 A. Abbas, M. J. Linman and Q. Cheng, Biosens. Bioelectron., 2012, 26, 1815-1824.

25 R. V. Nair, K. Dileep, V. S. Gummaluri and C. Vijayan, Plasmonics, 2017, 12, 1649-1656.

26 R. V. Nair, A. Prakash and C. Vijayan, J. Phys. D. Appl. Phys., 2018, 51, 04510.

27 X. Zhao, T. Huang, P. S. Ping, X. Wu, P. Huang, J. Pan, Y. Wu and Z. Cheng, Sensors, 2018, 18(7), 2056.

28 S. Raveendran, H. T. Lim, T. Maekawa, M. Vadakke Matham and D. Sakthi Kumar, Nanoscale, 2018, 10, 13959-13968.

29 E. Helmerhorst, D. J. Chandler, M. Nussio and C. D. Mamotte, Clin. Biochem. Rev., 2012, 33, 161-173.

30 D. Hao, C. Hu, J. Grant, A. Glidle and D. R. S. Cumming, Biosens. Bioelectron., 2018, 100, 23-27.

31 Y. H. Liao, Y. J. Chang, Y. Yoshiike, Y. C. Chang and Y. R. Chen, Small, 2012, 8, 3631-3639.

32 P. A. T. Martins, S. Alsaiari, K. Julfakyan, Z. Nie and N. M. Khashab, Chem. Commun., 2017, 53, 2102-2105.

33 Y. Xia, P. Padmanabhan, S. Sarangapani, B. Gulyás and M. Vadakke Matham, Sci. Rep., 2019, 9, 1-11.

34 K. V. Sreekanth, J. K. Chua and V. M. Murukeshan, Appl. Opt., 2010, 49, 6710-6717.
35 Z. Li, K. Sun, Z. Du, B. Chen and X. He, Nanomaterials, 2018, 8, 265.

36 X. Wang, D. P. Yang, P. Huang, M. Li, C. Li, D. Chen and D. Cui, Nanoscale, 2012, 4, 7766-7772.

37 Y. H. Su, S. L. Tu, S. W. Tseng, Y. C. Chang, S. H. Chang and W. M. Zhang, Nanoscale, 2010, 2, 2639-2646.

38 Y. M. Sabri, A. E. Kandjani, S. J. Ippolito and S. K. Bhargava, Sci. Rep., 2016, 6, 1-10.

39 M. Azimzadeh, N. Nasirizadeh, M. Rahaie and H. NaderiManesh, RSC Adv., 2017, 7, 55709-55719.

40 Z. Aghili, N. Nasirizadeh, A. Divsalar, S. Shoeibi and P. Yaghmaei, Biosens. Bioelectron., 2017, 95, 72-80.

41 L. Chen, Y. Huang, T. T. Xing, L. Ge, T. Yang, B. Chen and C. Z. Huang, J. Mater. Chem. C, 2017, 5, 7806-7812.

42 E. A. Elbassal, C. Morris, T. W. Kent, R. Lantz, B. Ojha, E. P. Wojcikiewicz and D. Du, J. Phys. Chem. C Nanomater. Interfaces, 2017, 121(36), 20007-20015.

43 P. C. Ke, E. H. Pilkington, Y. Sun, I. Javed, A. Kakinen, G. Peng, F. Ding and T. P. Davis, Adv. Mater., 2019, 1901690.

44 N. Xiong, Y. Zhao, X. Dong, J. Zheng and Y. Sun, Small, 2017, 13, 1-14.

45 G. Plascencia-Villa, A. Ponce, J. F. Collingwood, M. Josefina Arellano-Jiménez, X. Zhu, J. T. Rogers, I. Betancourt, M. José-Yacamán and G. Perry, Sci. Rep., 2016, 6, 1-12.

46 A. L. Clos, R. Kayed and C. A. Lasagna-Reeves, Front. Neurol., 2012, 1-10.

47 M. N. Sabbagh, J. Shi, M. Lee, L. Arnold, Y. Al-Hasan, J. Heim and P. McGeer, BMC Neurol., 2018, 18, 155.

48 Y. K. Yoo, J. Kim, G. Kim, Y. S. Kim, H. Y. Kim, S. Lee, W. W. Cho, S. Kim, S. M. Lee, B. C. Lee, J. H. Lee and K. S. Hwang, Sci. Rep., 2017, 7, 1-10.

49 J. K. Tee, L. X. Yip, E. S. Tan, S. Santitewagun, A. Prasath, P. C. Ke, H. K. Ho and D. T. Leong, Chem. Soc. Rev., 2019, 48, 5381-5407.

50 L. P. Jameson, N. W. Smith and S. V. Dzyuba, ACS Chem. Neurosci., 2012, 3, 807-819. 